\title{
Protective effect of nebivolol on gentamicin-induced nephrotoxicity in rats
}

\author{
Dursun $\mathrm{M}^{1}$, Sahin $\mathrm{S}^{2}$, Besiroglu $\mathrm{H}^{3}$, Otunctemur $\mathrm{A}^{3}$, Ozbek E $\mathrm{E}^{4}$, Cakir $\mathrm{SS}^{3}$, Cekmen $\mathrm{M}^{5}$, Somay $\mathrm{A}^{6}$
}

Bahcelievler State Hospital, Department of Urology, Istanbul, Turkey. mrt_drsn@hotmail.com

\begin{abstract}
OBJECTIVE: Nephrotoxicity is a major complication of gentamicin (GEN), which is widely used in the treatment of severe Gram-negative infections. As we know, treatment with nebivolol has been shown to decrease renal fibrosis and glomerular injury as well as improve endothelial dysfunction. Therefore, we evaluated the potential protective effect of nebivolol (NBV) against GEN-induced nephrotoxicity in rats.

MATERIAL AND METHOD: Twenty-four rats were randomly divided into four groups: control group (Group 1); rats intraperitoneally injected with GEN (100 mg/kg/day; Group 2); rats treated with GEN plus distilled water (Group 3); and rats treated with GEN plus NBV (10 mg/kg/day; Group 4). After 15 days, the rats were sacrificed, their kidneys taken, and blood analysis performed. Tubular necrosis and interstitial fibrosis scores were determined histopathologically in a part of kidneys; nitric oxide (NO), malondialdehyde (MDA), and reduced glutathione (GSH) levels were determined in other part of kidneys.

RESULTS: The GSH levels in renal tissue of only GEN-treated rats were significantly lower than those in control group, and administration of NBV to GEN-treated rats significantly increased the level of GSH. The group that was given GEN and NBV had significantly lower MDA and NO levels in kidney cortex tissue than that given GEN alone. Despite the presence of mild tubular degeneration, the rats treated with GEN+NBV showed a less severe tubular necrosis, and their glomeruli maintained a better morphology compared to GEN group.

CONCLUSION: NBV exerts antioxidant, anti-inflammatory and antifibrotic effects on GEN-induced kidney damage by reducing oxidative stress in rat model (Tab. 3, Fig. 2, Ref. 68). Text in PDF www.elis.sk.

KEY WORDS: nebivolol, rat, gentamicin, oxidative stress, nephrotoxicity.
\end{abstract}

\section{Introduction}

The kidney is a vital organ in health and disease. The main function of the kidneys is to maintain total body fluid volume, its composition, and acid-base balance. Many environmental contaminants and chemical variables, including drugs, alter the functions of the kidneys $(1,2)$. Among the classes of aminoglycoside antibiotics, gentamicin (GEN) is efficacious and commonly used in clinical practice for the treatment of life-threatening Gram-negative bacterial infections $(3,4)$. However, the usefulness of GEN is limited in clinical practice by the development of nephrotoxicity. GEN causes drug-induced nephrotoxicity by a direct dosedependent mechanism in $30 \%$ of patients treated for more than 7 days $(5,6)$. In some cases, this side effect is so severe that the use of the drug must be discontinued. Despite introduction of newer

${ }^{1}$ Bahcelievler State Hospital, Department of Urology, Istanbul, Turkey, ${ }^{2}$ Bilecik State Hospital, Department of Urology, Bilecik, Turkey, ${ }^{3} \mathrm{Okmey}-$ dani Training and Research Hospital, Department of Urology, Istanbul, Turkey, ${ }^{4}$ Istanbul University, Cerrahpasa Medical Faculty, Department of Urology, Istanbul, Turkey, ${ }^{5}$ Kocaeli University Medical Faculty, Department of Biochemistry, Kocaeli, Turkey, and ${ }^{6} \mathrm{Fatih}$ Sultan Mehmet Training and Research Hospital, Department of Pathology, Istanbul, Turkey

Address for correspondence: M. Dursun, Dr, Bahcelievler State Hospital, Department of Urology, 34180, Kocasinan Merkez, Bahcelievler, Istanbul, Turkey. Phone/Fax: +90.212 .4967000$ and less toxic antibiotics, GEN is still used clinically because of its rapid bactericidal action, broad-spectrum activity, clinical effectiveness, synergism with beta-lactam antibiotics and low cost (7). Most aminoglycosides are excreted in urine after drug administration; approximately $5-10 \%$ of the dose accumulates in the renal cortex and remains there long after discontinuation of the drug $(8,9)$. GEN is essentially eliminated by glomerular filtration and partially reabsorbed by proximal tubular cells (10). The specificity of GEN for renal toxicity is apparently related to its preferential accumulation in the renal proximal convoluted tubules, reaching a concentration of 5-50 times higher than that in plasma in the tubular renal cell (11).

Nephrotoxicity induced by GEN is a complex phenomenon characterized by an increase in plasma creatinine and urea levels and severe proximal renal tubular necrosis followed by deterioration and renal failure $(6,12)$. Lysosomes are the first and obvious sites of sequestration and accumulation of GEN in proximal tubules. This accumulation induces the process of lysosomal phospholipidosis, which is a key pathological mechanism contributing to renal toxicity resulting in tubular necrosis $(13,14)$. Gentamicin-induced tubular necrosis stimulates inflammatory events by recruiting intercellular adhesion molecule (ICAM)-1 and monocyte chemoattractant protein (MCP-1) at the site of injury, which enhances the migration of monocytes and macrophages to the site of tissue damage, thus ultimately leading to renal pathogenesis 
$(15,16)$. The inflammatory and fibrogenic responses to gentamicin were associated with the increases in transforming growth factor (TGF-B), nuclear factor kappa B (NF-KB), endothelin, and angiotensin II levels, thus implying their involvement in the progression of tubulointerstitial nephritis (17). Reactive oxygen species (ROS) are considered to be important mediators of GEN nephrotoxicity (18-20). In vitro and in vivo studies have shown that GEN enhances the generation of ROS metabolites, which may cause cellular injury and necrosis via several mechanisms, including peroxidation of membrane lipids, protein oxidation, and DNA damage (21). This elevation of ROS would stimulate the activation or expression of proinflammatory and proapoptotic mediators, including nuclear factor kappa B (NF-KB), leukocyte adhesion molecules and TGF-B contributing to kidney damage induced by gentamicin (22-24).

The role of nitric oxide (NO) in the pathophysiology of GEN-induced rat nephrotoxicity has been already studied (25). NO is a highly diffusible free radical gas produced by NO synthase (NOS) of which three isoforms have been identified: neural NOS (nNOS), endothelial NOS (eNOS), and inducible NOS (iNOS) (26). Since inducible NOS (iNOS) is responsible for the production of sustained high levels of NO, it is often considered the major cause of autotoxicity under oxidative stress. Under simultaneous generation of $\mathrm{NO}$ and ROS, the cellular antioxidant capabilities are also suppressed (27-29). It has been reported that activation of inducible NOS (iNOS) aggravates renal injury in GEN-associated rat nephrotoxicity (25).

Nebivolol (NBV) is a third generation selective $\beta-1$ adrenergic receptor blocker with additional vasodilating properties that is currently used in the therapy of hypertension and coronary heart disease $(30,31)$. These vasodilating properties can be attributed to an endothelial release of NO, possibly due to a $\beta 3$ adrenoceptor mediated stimulation of $\operatorname{eNOS}(32,33)$. Nebivolol has also been shown to possess a direct scavenging activity on oxygen radicals with consequent potent antioxidant properties in animal and human studies (34-36). Limited studies have shown that systemic treatment with this beta-blocker increases renal tissue levels of bioavailable NO and reduces renal fibrosis (37-39).

Therefore, the aim of the present study was to find out whether NBV could prevent GEN-induced nephrotoxicity in rats.

\section{Materials and metdods}

\section{Animals}

Adult male Wistar albino rats (200-250 g) were housed in clean plastic cages at room temperature and in a humidity-controlled facility with a constant 12-h light/dark cycle with free access to food and water. The use of animals and experimental protocol were approved by the Institutional Animal Care and Use Committee, and animals were treated in accordance with the Guide for the Care and Use of Laboratory Animals of Research Council.

\section{Drugs}

GEN was purchased from Bilim Pharmaceuticals (Istanbul, Turkey), and NBV was purchased from Ulugaylar Pharmaceuti- cals (Istanbul, Turkey). GEN was dissolved in saline and injected intraperitoneally. NBV diluted with saline to a final concentration of $10 \mathrm{mg} / \mathrm{kg}$ was administered by nasogastric gavage.

\section{Treatment and experimental design}

After a quarantine period of seven days, twenty-four rats were randomly divided into four groups, each consisting of six animals as follows: control rats (Group 1); rats intraperitoneally injected with GEN for 14 consecutive days (100 mg/ kg/day; Group 2); rats treated with GEN plus distilled water (vehicle) via nasogastric gavage for 14 days (Group 3); rats treated with GEN plus NBV $(10 \mathrm{mg} / \mathrm{kg} /$ day) for 14 days (Group 4). NBV was administered immediately after the injection of GEN. Rats were treated for 14 days. After 15 days, rats were killed, their kidneys taken, and blood analysis performed. We stored both parts of the kidneys in formaldehyde during analysis. Tubular necrosis and interstitial fibrosis scores were determined histopathologically in a part of kidneys; nitric oxide (NO), malondialdehyde (MDA), and glutathione (GSH) levels were determined in other part of the kidneys. Urea-creatinine, $\mathrm{Na}^{+}$, and $\mathrm{K}^{+}$levels were investigated as part of blood analysis.

\section{Biochemical analysis}

On day 15 , i.e. twenty-four hours after the administration of last doses of GEN and NBV, the rats were anesthetized by intraperitoneal injection of ketamine and sacrificed. Twenty-four-hour urine collections had been obtained in standard metabolic cages one day before the rats were killed. Renal cortical tissues were separated into two parts for biochemical analysis and light microscopic examination. Blood samples were also taken by cardiac puncture to assess the serum levels of urea, creatinine, $\mathrm{Na}$, and $\mathrm{K}$ concentrations. The tissue liquid was frozen with nitrogen and kept at $-80{ }^{\circ} \mathrm{C}$. In frozen tissues, MDA, end product of lipid peroxidation, GSH, nonenzymatic antioxidant, total nitrite, and stable product of $\mathrm{NO}$ were evaluated biochemically as the means of oxidative stress.

Renal impairment was assessed by serum urea and creatinine levels, as well as by kidney histology. Serum urea and creatinine levels were determined with an autoanalyzer (Syncron LX20, Ireland) by using commercial Becman Coulter diagnostic kits. Kidney tissue $(300 \mathrm{mg}$ ) was homogenized in ice-cold tamponade containing $150 \mathrm{mM} \mathrm{KCl}$ for determination of MDA. MDA levels were assayed for products of lipid peroxidation. MDA, referred to as thiobarbituric acid reactive substance, was measured with thiobarbituric acid at $532 \mathrm{~nm}$ in a spectrofluorometer, as described previously (40). Reduced GSH was estimated by the method of Moron et al (41). The color developed was read at $412 \mathrm{~nm}$. Protein concentrations in all samples were measured using the method of Lowry et al (42).

Total nitrite (NOx) was quantified by the Griess reaction (43) after incubating the supernatant with Escherichia coli nitrate reductase to convert $\mathrm{NO}_{3}$ to $\mathrm{NO}_{2}$. Griess reagent $(1 \mathrm{~mL} 1 \%$ sulfanilamide, $0.1 \%$ naphthylethylenediamine hydrochloride, and $2.5 \%$ phosphoric acid; Sigma Chemical Co., St. Louis, MO, USA) was then added to $1 \mathrm{~mL}$ of supernatant. The absorbance was read at 
$545 \mathrm{~nm}$ after a $30-\mathrm{min}$ incubation. The absorbance was compared with the standard graph of $\mathrm{NaNO}_{2}$, obtained from the reduction of $\mathrm{NaNO}_{3}(1-100 \mathrm{lmol} / \mathrm{L})$. The accuracy of the assay was checked in two ways, namely as inter- and intraassay coefficients of variation, which were $7.52 \%$ and $4.61 \%$, respectively. To check the conversion of nitrate to nitrite (recovery rate), the known amounts of nitrate were added to control plasma samples which were then deproteinized and reduced.

\section{Histopathological evaluation}

Histopathological evaluation of kidney tissues was performed. Paraffin-embedded specimens were cut into 6-mm-thick sections and stained with hematoxylin \& eosin and Masson's trichrome for examination under the light microscopic examination using a conventional protocol (BH-2; Olympus, Tokyo, Japan) (44). A semi-quantitative evaluation of renal tissues was accomplished by scoring the degree of severity according to previously published criteria. Tubular necrosis was evaluated in all kidney sections. (45). Briefly, a minimum of 50 proximal tubules associated with 50 glomeruli were examined for each slide and an average score was obtained. The severity of lesion was graded from 0 to 3 according to the percentage of tubular involvement. Slides were examined and assigned for severity of changes using scores on scale, in which (0) denotes no change, grade (1) change affecting $<25 \%$ tubular damage (mild), grade (2) change affecting 25-50 $\%$ of tubules (moderate), and grade (3) change affecting $>50 \%$ of tubules (severe; 46). To evaluate leukocyte infiltration, the widening of interstitial spaces with focal leukocyte infiltration was assessed in five randomly chosen sections prepared from each kidney sample. For each section, the average number of leukocytes per $0.28 \mathrm{~mm}$ was calculated from these leukocyteinfiltrated foci using a high-power microscopic field. To estimate the grade of interstitial fibrosis, the interstitial area stained green with Masson's trichrome was evaluated as a percentage of the total examined area in five randomly chosen sections prepared from each kidney sample using an image analyzer (Leica; Leica
Micros Imaging Solutions, Cambridge, UK). For each section, interstitial space widening with focal leukocyte infiltration and interstitial fibrosis was assessed in high-power fields to quantify the results (47).

\section{Statistical snalysis}

Results of all groups were shown as mean values \pm standard deviation (SD). Statistical analyses of the histopathologic evaluation of the groups were carried out by the chi-square test and biochemical data were analyzed by one-way analysis of variance (ANOVA). The significance between the two groups was determined by the Dunnett's multiple comparison test, while $\mathrm{p}<0.05$ was accepted as a statistically significant value.

\section{Results}

No deaths or remarkable signs of external toxicity were observed in the groups of rats given GEN either alone or in combination with NBV.

\section{Urine volume}

The 24-h urine volume in the GEN-treated group was significantly higher than in the control group $(\mathrm{p}<0.01)$, indicating the presence of GEN-induced polyuria, whereas in the group treated with GEN+NBV, it was not different from that of the control group, pointing out the protective role of NBV against acute tubular necrosis (Tab. 1).

\section{Biochemical variables in plasma and tissue}

$\mathrm{Na}^{+}$and $\mathrm{K}^{+}$concentrations were similar among the four groups. Serum urea and creatinine levels were significantly higher in rats treated with GEN alone than in control and GEN + NBV groups $(\mathrm{p}<0.01)$. Administration of NBV to GEN-treated rats caused a decrease in serum urea and creatinine levels (Tab. 1).

The GSH levels in renal tissue of only GEN-treated rats were significantly lower than those in control group $(\mathrm{p}<0.05)$, and ad-

Tab. 1. Effects of GEN alone and those of its combination with MK on plasma urea, creatinine, Na, K, and 24-h urine volume levels in rats.

\begin{tabular}{lcccc}
\hline Parameters & $\begin{array}{c}\text { Control } \\
\text { (Group 1) }\end{array}$ & $\begin{array}{c}\text { GEN } \\
\text { (Group 2) }\end{array}$ & $\begin{array}{c}\text { GEN+Ve } \\
\text { (Group 3) }\end{array}$ & $\begin{array}{c}\text { GEN + NBV } \\
\text { (Group 4) }\end{array}$ \\
\hline Urea $(\mathrm{mg} / \mathrm{dl})$ & $33 \pm 7.8$ & $107 \pm 13.9 \mathrm{a}$ & $105 \pm 13.4$ & $40.1 \pm 8.4 \mathrm{~b}$ \\
\hline Creatinine $(\mathrm{mg} / \mathrm{dl})$ & $0.42 \pm 0.1$ & $2.08 \pm 0.4 \mathrm{a}$ & $1.97 \pm 0.4$ & $0.68 \pm 0.2 \mathrm{~b}$ \\
\hline $\mathrm{Na}(\mathrm{mmol} / \mathrm{L})$ & $138.9 \pm 1.3$ & $139.9 \pm 2.5$ & $139.3 \pm 2.2$ & $139.2 \pm 2.1$ \\
\hline $\mathrm{K}(\mathrm{mmol} / \mathrm{L})$ & $3.88 \pm 0.2$ & $4.1 \pm 0.5$ & $4.04 \pm 0.4$ & $3.9 \pm 0.3$ \\
\hline 24-h urine volume $(\mathrm{mL})$ & $8.9 \pm 1.2$ & $21.2 \pm 4.1 \mathrm{a}$ & $20.1 \pm 3.2$ & $9.2 \pm 1.4 \mathrm{~b}$ \\
\hline
\end{tabular}

Values are expressed as mean \pm SD for six rats in each group. Groups: Control, GEN (gentamicin), GEN + Ve (gentamicin + vehicle), GEN + NBV (gentamicin + nebivolol).

a Significantly different from control. ${ }^{\mathrm{b}}$ Significantly different from gentamicin group $(\mathrm{p}<0.001)$

Tab. 2. Effects of NBV on rat kidney: NO, MDA, and GSH levels.

\begin{tabular}{|c|c|c|c|c|}
\hline Parameters & $\begin{array}{c}\text { Control } \\
\text { (Group 1) }\end{array}$ & $\begin{array}{c}\text { GEN } \\
\text { (Group 2) }\end{array}$ & $\begin{array}{l}\text { GEN+Ve } \\
\text { (Group 3) }\end{array}$ & $\begin{array}{c}\text { GEN + NBV } \\
\text { (Group 4) }\end{array}$ \\
\hline NO (nmol/g wet tissue) & $29.8 \pm 8.5$ & $47.6 \pm 7.2^{\mathrm{a}}$ & $44.9 \pm 5.7$ & $30.3 \pm 7.9^{\mathrm{b}}$ \\
\hline MDA (nmol/g wet tissue) & $2.6 \pm 0.7$ & $4.2 \pm 1.1^{\mathrm{a}}$ & $3.9 \pm 1.1$ & $2.7 \pm 0.9^{\mathrm{b}}$ \\
\hline GSH (umol/g wet tissue) & $2.4 \pm 0.9$ & $1.4 \pm 0.5^{\mathrm{a}}$ & $1.3 \pm 0.6$ & $2.7 \pm 0.5^{\mathrm{b}}$ \\
\hline
\end{tabular}

Values are expressed as mean \pm SD for six rats in each group. NO, nitric oxide; MDA, malondialdehyde; GSH, glutathione. ${ }^{\text {a }}$ Significantly different from control. ${ }^{\text {b Signifi- }}$ cantly different from gentamicin group $(\mathrm{p}<0.05)$ 

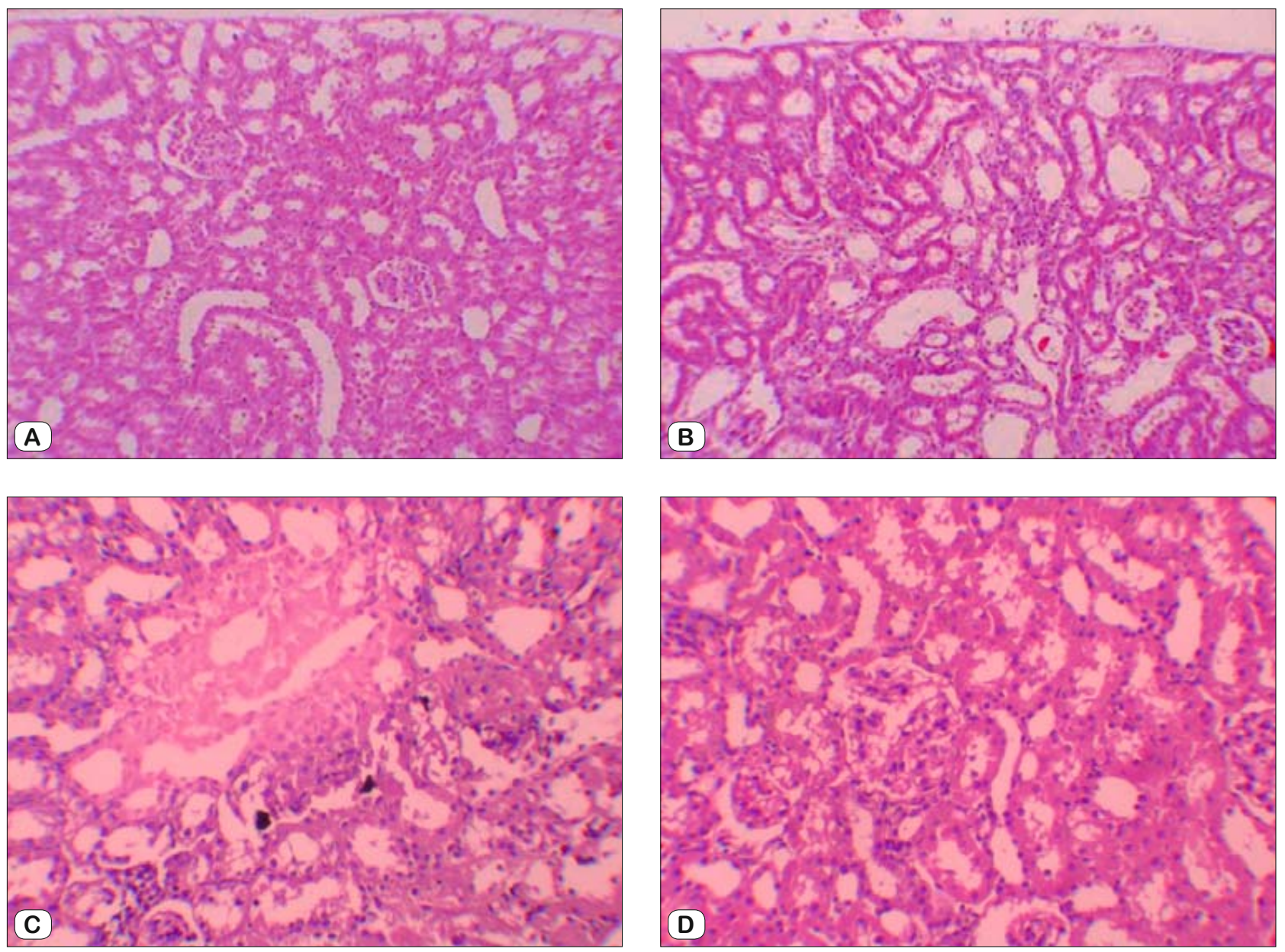

Fig. 1. (A) Normal tubulus and glomerules in kidney cortex H\&E $100 \times$ (control group). (B) Severe tubular necrosis, tubular degeneration, and epithelial vacuolization in the proximal tubules H\&E $100 \times$ (GEN-reated group). (C) Moderate tubular necrosis, tubular degeneration, and epithelial vacuolization in the proximal tubules H\&E $100 \times($ GEN + vehicle treated group). (D) Mild epithelial granulovacuolization in the proximal tubules and normal glomerules H\&E $100 \times($ GEN + NBV treated group).

ministration of NBV to GEN-treated rats significantly increased the level of GSH ( $\mathrm{p}<0.05)$ (Tab. 2). The group given GEN and NBV had significantly lower MDA levels in kidney cortex tissue than those given GEN alone. There was high level of NO in GENtreated group; however, NO levels in group treated with GEN + NBV were significantly lower than those in GEN-treated group (Tab. 2). There was no significant difference in biochemical variables between NBV and control groups (Tabs 1 and 2).

\section{Histopathologic examination results}

Histopathologic examination of kidney showed that there were no pathologic features in the control group (Fig. 1A). Compared to control group, rats treated with GEN and GEN + vehicle yielded mild and severe tubular necrosis, tubular degeneration, and epithelial vacuolization in the proximal tubules (Figs $1 \mathrm{~B}$ and 1C). Despite the presence of mild tubular degeneration and epithelial vacuolization in their proximal tubules, the tubular necrosis in

Tab. 3. Semiquantitative analysis of tubular necrosis, interstitial fibrosis, leukocyte infiltration in control, GEN, GEN + Ve and GEN+ NBV groups.

\begin{tabular}{|c|c|c|c|c|c|c|c|c|c|c|c|c|c|}
\hline & \multirow[b]{2}{*}{$\mathrm{n}$} & \multicolumn{4}{|c|}{ Tubular necrosis } & \multicolumn{4}{|c|}{ Interstitial fibrosis } & \multicolumn{4}{|c|}{ Leukocyte infiltration } \\
\hline & & 0 & 1 & 2 & 3 & 0 & 1 & 2 & 3 & 0 & 1 & 2 & 3 \\
\hline Control & 6 & 6 & 0 & 0 & 0 & 6 & 0 & 0 & 0 & 5 & 1 & 0 & 0 \\
\hline $\mathrm{GEN}^{\mathrm{a}}$ & 6 & 0 & 3 & 2 & 1 & 3 & 3 & 0 & 0 & 0 & 3 & 3 & 0 \\
\hline $\mathrm{GEN}+\mathrm{Ve}$ & 6 & 0 & 4 & 2 & 0 & 4 & 2 & 0 & 0 & 0 & 3 & 3 & 0 \\
\hline $\mathrm{GEN}+\mathrm{NBV}^{\mathrm{b}}$ & 6 & 0 & 6 & 0 & 0 & 6 & 0 & 0 & 0 & 3 & 3 & 0 & 0 \\
\hline
\end{tabular}

Score 0 : no degeneration, 1: mild degeneration, 2: moderate degeneration, 3: severe degeneration

${ }^{a}$ Statistically significantly different from control (including tubular necrosis and leukocyte infiltration)

${ }^{\mathrm{b}}$ Statistically significantly different from GEN and $\mathrm{P}<0.05$ (including tubular necrosis and leukocyte infiltration) 

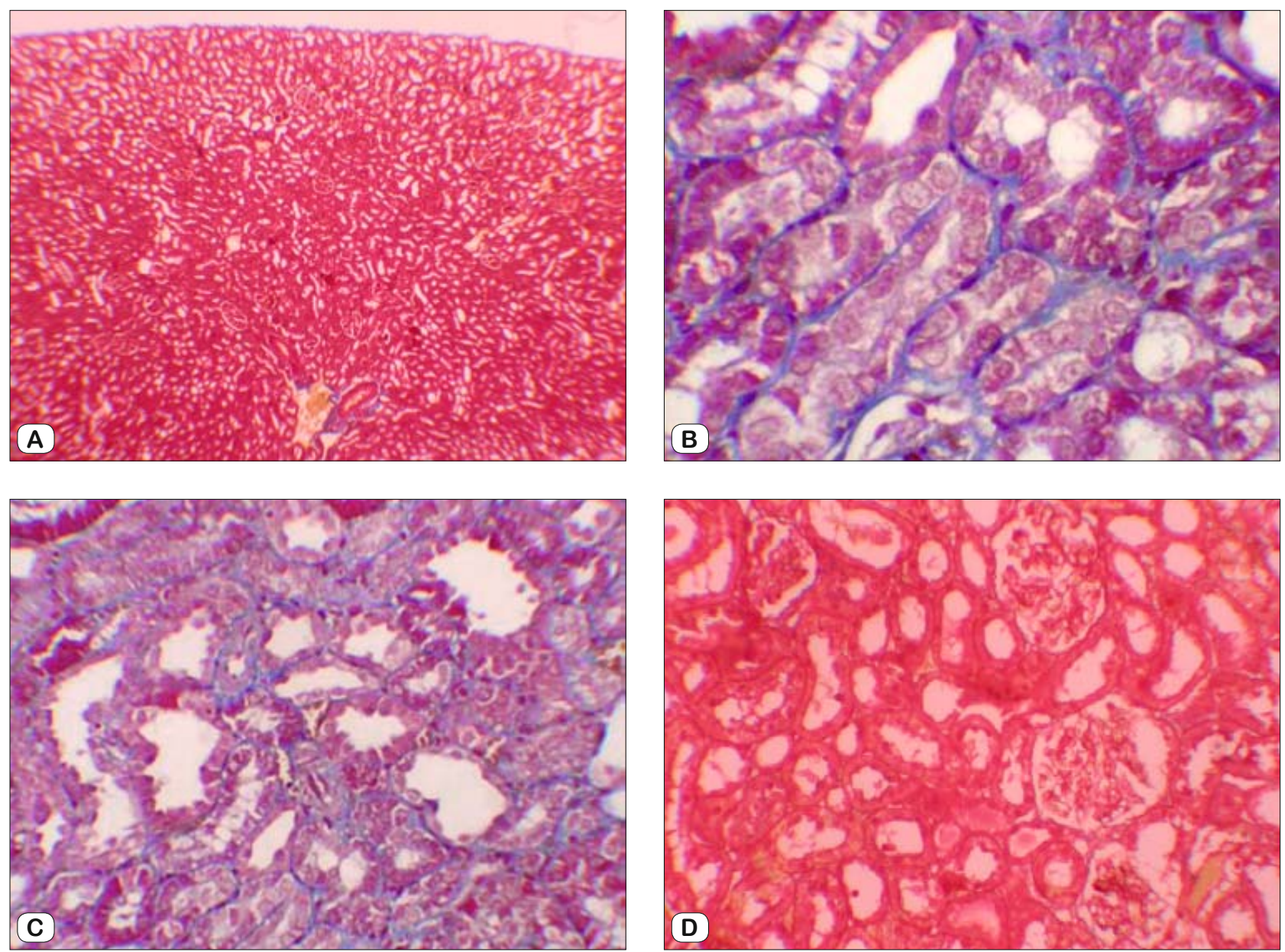

Fig. 2. (A) No fibrosis in control group, staining with Masson's trichrome $100 \mathrm{X}$ (B) Mild fibrosis in interstitium, staining with Masson's trichrome $400 \mathrm{X}$ (GEN-treated group). (C) Mild fibrosis in interstitium, staining with Masson's trichrome $200 \mathrm{X}$ (GEN + vehicle treated group). (D) No fibrosis in GEN + NBV treated group, staining with Masson's trichrome 100X.

rats treated with GEN + NBV was less severe, and their glomeruli maintained a better morphology compared with GEN group (Fig. 1D). These changes are summarized in Table 3. After staining with Masson trichrome, no statistical difference was found between groups in kidney fibrosis scores (Tab. 3, Figs 2A-2D). There was no significant difference in histopathologic results of kidney between NBV and control group (Tab. 3).

\section{Discussion}

Kidneys are easily susceptible to damage from drugs because of larger perfusion and increased concentration of excreted compounds occurring in renal tubular cells during absorption and secretion. Aminoglycoside antibiotics are well known to cause serious nephrotoxicity that limits its clinical use. GEN is a widely used aminoglycoside antibiotic to treat serious and life-threatening Gram-negative bacterial infections and has been shown to cause marked histological damage, in particular to renal proximal convoluted tubules. The damage results in swelling, vacuolization and necrosis of epithelial cells $(48,49)$. This could be due to the fact that proximal convoluted tubules are the primary sites of reabsorption and active transport, which leads to a higher concentration of gentamicin in the epithelial lining of these tubules. This might also suggest that gentamicin toxicity is related to its accumulation in the proximal tubules (8).

Gentamicin is known to generate reactive oxygen species associated with an increase in lipid peroxidation and decrease in antioxidant enzyme activity in the kidney (18). Results of many studies have shown that altered concentrations of various biochemical indicators of oxidative stress in kidney tissue are due to GEN (50). Because of the obvious responsibility of ROS in GEN-induced renal damage, several antioxidant agents have been used effectively to ameliorate GEN-induced nephrotoxicity $(3,6)$. Several investigators reported that treatment with GEN produces nephrotoxicity, evident by the reduction in renal functions which is characterized by an increase in BUN and serum creatinine levels (51). GEN nephrotoxicity is clinically important to the extent that nephrotoxicity is typically associated with nonoliguric or even polyuric acute renal failure, that is with azotemia in the presence of urine output in amount of 1-2 L/day (8). In present study, the 
24-h urine volume in the GEN group was significantly higher than in the control group, thus indicating the presence of GEN-induced polyuria, whereas in GEN+NBV group, it was not different from that of the control group, thus suggesting the protective effect of NBV against acute tubular necrosis. Also, increased serum urea and creatinine levels in GEN-treated rats reflect the renal injury. In contrast to the previous studies, serum $\mathrm{K}^{+}$levels were similar between GEN-induced nephrotoxicity and control groups. The final studies show that $\mathrm{K}^{+}$levels were not increased in rats with GEN-induced nephrotoxicity $(52,53)$. Administration of NBV protects the kidney function from GEN as indicated by preventing an increase in serum urea and creatinine levels. NBV repairs the renal function by preserving the structural integrity of renal cells against GEN challenge.

NO plays a crucial role in kidney angiogenesis by ameliorating ischemia and hypoxia in the renal interstitial area and slowing the progression of interstitial fibrosis (54). Excessive production of NO by iNOS has been associated with nitrosative stress or interactions with ROS that form peroxynitrites which are very cytotoxic oxidant radical species. Under the simultaneous generation of $\mathrm{NO}$ and ROS, the cellular antioxidant capabilities are also suppressed (55). Peroxynitrite anion formation, protein tyrosine nitration, and hydroxyl radical production may be responsible for the evolution of renal injury induced by GEN (56). Previous studies have demonstrated that $\mathrm{NO}$ and peroxynitrites play an important role in acute renal failure caused by GEN (57). Beneficial effects of selective iNOS blockade in GEN-induced nephrotoxicity have been investigated and it was found out that the inhibition of iNOS may prevent GEN-induced nephrotoxicity, whereas non-selective inhibition of NOS aggravates it (25). NBV increases NO production by activating eNOS, which may have a beneficial role as a vasodilator by inducing an increase in renal blood flow and glomerular filtration $(58,59)$. On the other hand, NBV directly interacts with free radicals, which reduces oxidative stress while the inflammatory cytokine production is inactivated. Consequently, the iNOS activity and production of toxic NO derived from iNOS are suppressed (60). Co-administration of NBV effectively suppressed the iNOS expression, which further confirms its ameliorative role against GEN-induced nephrotoxicity. TGF- $\beta$ and nuclear factor$\mathrm{kB}$ are fibrogenic, proinflammatory and proapoptotic cytokines that are involved in GEN-induced renal damage. NF-kB is activated during increased oxidative stress and expression of iNOS, causing interstitial fibrosis $(46,61)$. TGF- $\beta$ is believed to play a role in the initiation and progression of renal fibrosis. TGF- $\beta$ was predominantly expressed by renal intrinsic cells and infiltrated inflammatory cells. Some studies have reported that TGF- $\beta$ contributes to fibrosis mainly through the Smad signaling pathway. However, increased NO derived from eNOS suppresses TGF- $\beta$ / Smad transactivation in endothelial cells $(62,63)$. It was shown that NBV treatment prevents renal fibrosis by reducing the rise of iNOS expression, oxidative stress parameters, and increasing NO derived from eNOS against GEN-induced nephrotoxicity.

A relationship between oxidative stress and nephrotoxicity has been well demonstrated in many experimental animal models. GEN-induced ROS are essential mediators of its nephrotoxic ef- fects causing cellular injury via several mechanisms including the peroxidation of membrane lipids, and oxidative damage of proteins and DNA (64). Therefore, herein we measured MDA, GSH, and nitric oxide (NO) as the means of oxidative stress. In the present study, MDA levels, i.e. the indicators of lipid peroxidation of the membranes as a result of oxidative damage (65), were significantly increased after GEN treatment, thus again demonstrating tissue damage. In parallel, GSH levels were decreased in renal tissue. $\mathrm{GSH}$, the main intracellular non-protein sulfhydryl, plays an important role in the maintenance of protein and lipid integrity, and provides major protection in oxidative injury by participating in the cellular defense systems against oxidative damage (66). In the group treated with GEN+NBV, we found decreased MDA levels and increased GSH levels. On the other hand, the renal NO levels in rats treated only with GM were found to be significantly higher than in those from the control group while the treatment with NBV significantly prevented the elevation of NO levels in our study. These findings strongly indicate that NBV is important in protecting the kidney from GEN-induced injury through improving the oxidant status. Animal models of aminoglycoside nephrotoxicity present residual areas of interstitial fibrosis in the renal cortex and progressive tubular injury (67). In this study, the histopathologic examination of the kidneys in GEN-treated rats showed severe and extensive damage in form of tubular necrosis and edema. This could be due to the formation of highly reactive radicals in consequence of oxidative stress caused by GEN. On the other hand, the tubules in rats from the GEN+ NBV group were nearly normal in their histological appearance except for a slight desquamation and atrophy of the tubular epithelial cells. Similar changes were also reported by some studies which demonstrated structural changes in renal tissue of gentamicin-treated animals and its reversal by various agents $(10,68)$. The results reported herein indicate that NBV exerts antioxidant, anti- inflammatory and antifibrotic effects on GEN-induced kidney damage in rat model by reducing oxidative stress. NBV is a high free-radical scavenger agent and offers protection against GEN-induced acute renal failure.

\section{References}

1. Mahmood I, Waters DH. A comparative study of uranyl nitrate and cisplatin induced renal failure in rat. Eur J Drug Metab Pharmacol 1994; 19: 327-336.

2. Begg EJ, Barclay ML. Aminoglycisides - 50 years on. Br J Clin Pharmacol 1995; 39: 597-603.

3. Parlakpinar H, Sahna E, Acet A, Mizrak B, Polat A. Effect of caffeic acid phenethyl ester (CAPE) on myocardial ischemia reperfusion induced apoptotic cell death. Toxicol 2005; 207: 169-177.

4. Balakumar P, Rohilla A, Thangathirupathi A. Gentamicin-induced nephrotoxicity: do we have a promising therapeutic approach to blunt it? Pharmacol Res 2010; 62: 179-186.

5. Rougier F, Claude D, Maurin M, Maire P. Aminoglycoside nephrotoxicity. Curr Drug Targets 2004; 4 (2): 153-162.

6. Cuzzocrea S, Mazzon E, Dugo $L$ et al. A role for superoxide in gentamicin-mediated nephropathy in rats. Eur J Pharmacol 2002; 450: 67-76. 
7. Edson RS, Terrell CL. The aminoglycosides. Mayo Clinic Proceedings 1999; 74 (5): 519-528.

8. Mingeot-Leclercq MP, Tulkens PM. Aminoglycosides: nephrotoxicity. Antimicrob Agents Chemother 1999; 43: 1003-1012.

9. Nagai J, Takano M. Molecular aspects of renal handling of aminoglycosides and strategies for preventing the nephrotoxicity. Drug Metab Pharmacokinet 2004; 19: 159-170.

10. Karadeniz A, Yildirim A, Simsek N, Kalkan Y, Celebi F. Spirulina platensis protects against gentamicin-induced nephrotoxicity in rats. Phytother Res 2008; 22: 1506-1510.

11. Abdel-Raheem IT, Abdel-Ghany AA, Mohamed GA. Protective effect of quercetin against gentamicin-induced nephrotoxicity in rats. Biol Pharm Bull 2009; 32: 61-67.

12. Al-Majed AA, Mostafa AM, Al-Rikabi AC, Al-Shabanah OA. Protective effects of oral arabic gum administration on gentamicin-induced nephrotoxicity in rats. Pharmacol Res 2002; 46 (5): 445-451.

13. Servais H, Van Der Smissen P, Thirion G et al. Gentamicin-induced apoptosis in LLC-PK1 cells: involvement of lysosomes and mitochondria. Toxicol Appl Pharmacol 2005; 206: 321-333.

14. Pedraza-Chaverri J, Barrera D, Maldonado PD et al. S-allylmercaptocysteine scavenges hydroxyl radical and singlet oxygen in vitro and attenuates gentamicin-induced oxidative and nitrosative stress and renal damage in vivo. BMC Clin Pharmacol 2004; 4: 5.

15. Geleilete TJ, Melo GC, Costa RS et al. Role of myofibroblasts, macrophages, transforming growth factor-beta endothelin, angiotensin-II, and fibronectin in the progression of tubulointerstitial nephritis induced by gentamicin. J Nephrol 2002; 15: 633-642.

16. Tang WW, Feng L, Mathison JC, Wilson CB. Cytokine expression, upregulation of intercellular adhesion molecule-1, and leukocyte infiltration in experimental tubulointerstitial nephritis. Lab Invest 1994; 70: 631-638.

17. EI Mouedden M, Laurent G, Mingeot-Leclercq MP, Tulkens PM. Gentamicin-induced apoptosis in renal cell lines and embryonic rat fibroblasts. Toxicol Sci 2000; 56: 229-239.

18. Banday AA, Farooq N, Priyamvada S, Yusufi AN, Khan F. Timedependent effects of gentamicin on the enzymes of carbohydrate metabolism, brush border membrane and oxidative stress in rat kidney tissues. Life Sci 2008; 82: 450-459.

19. Polat A, Parlakpinar H, Tasdemir S et al. Protective role of aminoguanidine on gentamicin-induced acute renal failure in rats. Acta Histochem 2006; 108: 365-371.

20. Ali BH. Agents ameliorating or augmenting experimental gentamicin nephrotoxicity: Some recent research. Food Chem Toxicol 2003; 41: $1447-1452$.

21. Priuska EM, Schacht J. Formation of free radicals by gentamicin and iron and evidence for an iron/gentamicin complex. Biochem Pharmacol 1995; 50: 1749-1752.

22. Diamond JR, Kees-Folts D, Ding G, Frye JE, Restrepo NC. Macrophages, monocyte chemoattractant peptide-1, and TGF beta 1 in experimental hydronephrosis. Am J Physiol 1994; 266: F926-F933.

23. Pueyo ME, Gonzalez W, Nicoletti A et al. Angiotensin II stimulates endothelial vascular cell adhesion molecule-1 via nuclear factor-kappaB activation induced by intracellular oxidative stress. Arterioscler Thromb Vasc Biol 2000; 20: 645-651.
24. Tang WW, Feng L, Mathison JC, Wilson CB. Cytokine expression, upregulation of intercellular adhesion molecule-1, and leukocyte infiltration in experimental tubulointerstitial nephritis. Lab Invest 1994; 70: 631-638.

25. Ghaznavi R, Kadkhodaee M. Comparative effects of selective and non-selective nitric oxide synthase inhibition in gentamicin-induced rat nephrotoxicity. Arch Toxicol 2007; 81: 453-457.

26. Kone BC. Nitric oxide synthesis in the kidney: isoforms, biosynthesis and functions in health. Semin Nephrol 2004; 24: 299-315.

27. Bogdan C. The multiplex function of nitric oxide in (auto) immunity. J Exp Med 1998; 187: 1361-1365.

28. De Pinto MC, Tommasi F, De Gara $L$ et al. Changes in the antioxidant systems as part of the signaling pathway responsible for the programmed cell death activated by nitric oxide and reactive oxygen species in tobacco Bright-Yellow 2 cells. Plant Physiol 2002; 130: 698-708.

29. Zhang C, Walker LM, Mayeux PR. Role of nitric oxide in lipopolysaccharide-induced oxidant stress in the rat kidney. Biochem Pharmacol 2000; 59: 203-209.

30. Gupta S, Wright HM. Nebivolol: a highly selective beta1-adrenergic receptor blocker that causes vasodilation by increasing nitric oxide. Cardiovasc Ther 2008; 26: 189-202.

31. Maffei A, Lembo G. Nitric oxide mechanisms of nebivolol. Ther Adv Cardiovasc Dis 2009; 3: 317-327.

32. Dessy C, Saliez J, Ghisdal $\mathbf{P}$ et al. Endothelial $\beta-3$ adrenoceptors mediate nitric oxide-dependent vasorelaxation of coronary microvessels in response to the third-generation $\beta$-blocker nebivolol. Circulation 2005; 112: 1198-1205.

33. Mason RP, Kalinowski L, Jacob RF, Jacoby AM, Malinski T. Nebivolol reduces nitroxidative stress and restores nitric oxide bioavailability in endothelium of black Americans. Circulation 2005; 112: 3795-3801.

34. de Groot AA, Mathy MJ, van Zwieten PA, Peters SL. Antioxidant activity of nebivolol in the rat aorta. J Cardiovasc Pharmacol 2004; 43 : $148-153$.

35. Mason RP, Kubant R, Jacob RF et al. Effect of nebivolol on endothelial nitric oxide and peroxynitrite release in hypertensive animals: role of antioxidant activity. Cardiovasc Pharmacol 2006; 48: 862-869.

36. Oelze M, Daiber A, Brandes RP et al. Nebivolol inhibits superoxide formation by NADPH oxidase and endothelial dysfunction in angiotensin II-treated rats. Hypertension 2006; 48: 677-684.

37. Kalinowski L, Dobrucki LW, Szczepanska- Konkel M et al. Thirdgeneration betablockers stimulate nitric oxide release from endothelial cells through ATP efflux: a novel mechanism for antihypertensive action. Circulation 2003; 107: 2747-2752.

38. Georgescu A, Pluteanu F, Flonta ML et al. The cellular mechanisms involved in the vasodilator effect of nebivolol on the renal artery. Eur $\mathrm{J}$ Pharm 2005; 508: 159-166.

39. Pires MJ, Rodríguez-Peña AB, Arévalo M et al. Long-term nebivolol administration reduces renal fibrosis and prevents endothelial dysfunction in rats with hypertension induced by renal mass reduction. J Hypertens 2007; 25: 2486-2489.

40. Wasowicz W, Neve J, Peretz A. Optimized steps in fluorometric determination of thiobarbituric acid reactive substances in serum: Importance of extraction $\mathrm{pH}$ and influence of sample preservation and storage. Clin Chem 1993; 39: 2522-2528. 
41. Moron MS, Depierre JW, Mannervik B. Levels of glutathione, glutathione reductase and glutathione S-transferase activities in rat lung and liver. Biochim Biophys Acta 1979; 582: 67-78.

42. Lowry OH, Rosenbrough NJ, Farr AL et al. Protein measurement with the Folin phenol reagent. J Biol Chem 1951; 193: 265-275.

43. Granger DL, Taintor RR, Boockvar KS et al. Measurement of nitrate and nitrite in biological samples using nitrate reductase and Griess reaction. Methods Enzymol 1999; 268: 142-151.

44. Sun Y, Oberley LW, Li Y. A simple method for clinical assay of superoxide dismutase. Clin Chem. 1998; 34: 497-500.

45. Allen CT. Laboratory methods in histochemistry. In: Prophet EB, Mills B, Arrington JB, Sobin LH, eds. American Registry of Pathology. Washington DC: Armed Forces Institute of Pathology; 1992: 53.

46. Ozbek E, Ilbey YO, Ozbek M, Simsek A, Cekmen M, Somay A. Melatonin attenuates unilateral ureteral obstruction induced renal injury by reducing oxidative stress, İnos, MAPK, and NF-kB expression. J Endourol 2009; 23 (7): 1165-1173.

47. Ayyildiz A, Nuhoğlu B, Gülerkaya B, Caydere M, Ustün H, Germiyanoglu $\mathrm{C}$ et al. Effect of intraurethral mitomycin $\mathrm{C}$ on healing and fibrosis in rats with experimentally induced urethral stricture. Int J Urol 2004; 11: 1122-1126.

48. Abdel-Gayoum AA, Ali BH, Abdel-Razig AA, Bashir AA, Ghywarsha K. Effect of gentamicin-induced nephrotoxicity on some carbohydrate metabolic pathways in the rat renal cortex. Arch Toxicol 1994; 68: 643-647.

49. Ali BH, Bashir AA. Effect of fish oil treatment on gentamicin nephrotoxicity in rats. Anal Nutr Metab 1994; 38: 336-339.

50. Humes HD. Aminoglycoside nephrotoxicity. Kidney Int 1988; 33: 900-911.

51. Atessahin A, Karahan I, Yilmaz S, Ceribasi A, Princci I: The effect of manganese chloride on gentamicin-induced nephrotoxicity in rats. Pharmacol Res 2003; 48: 637-642.

52. Silan C, Uzun O, Comunoglu NU, Gokcen S, Bedirhan S, Cengiz M. Gentamicin-induced nephrotoxicity in rats ameliorated and healing effects of resveratrol. Biol Pharm Bull 2007; 30: 79-83.

53. Said MM. The protective effect of eugenol against gentamicininduced nephrotoxicity and oxidative damage in rat kidney. Fundam Clin Pharmacol 2011; 25: 708-716.

54. Sun D, Wang Y, Liu $C$ et al. Effects of nitric oxide on renal interstitial fibrosis in rats with unilateral ureteral obstruction. Life Sci 2012; 90: 900-909.
55. Andre M, Latado H, Felley-Bosco E. Inducible nitric oxide synthasedependent stimulation of PKGI and phosphorylation of VASP in human embryonic kidney cells. Biochem Pharmacol 2005; 69: 595-602.

56. Klahr S. The role of L-arginine in hypertension and nephrotoxicity. Curr Opin Nephrol Hypertens 1998; 7: 547-550.

57. Christo JS, Rodrigues AM, Mouro MG et al. Nitric oxide (NO) is associated with gentamicin (GENTA) nephrotoxicity and the renal function recovery after suspension of GENTA treatment in rats. Nitric Oxide 2011; 24: 77-83.

58. Tran Quang T, Rozec B, Audigane $\mathbf{L}$ et al. Investigation of the different adrenoceptor targets of nebivolol enantiomers in rat thoracic aorta. Br J Pharmacol 2009; 156: 601-608.

59. Maffei A, Vecchione $C$, Aretini A et al. Characterization of nitric oxide release by nebivolol and its metabolites. Am J Hypertens 2006; 19: $579-586$.

60. Evangelista S, Garbin U, Pasini AF et al. Effect of DL-nebivolol, its enantiomers and metabolites on the intracellular production of superoxide and nitric oxide in human endothelial cells. Pharmacol Res 2007; 55: 303-309.

61. Cheung RT, Tipoe GL, Tam S et al. Preclinical evaluation of pharmacokinetics and safety of melatonin in propylene glycol for intravenous administration. J Pineal Res 2006; 41: 337-343.

62. Schiffer M, von Gersdorff G, Bitzer M, Susztak K, Böttinger EP. Smad proteins and transforming growth factor-beta signaling. Kidney Int Suppl 2000; 77: S45-52.

63. Saura M, Zaragoza C, Herranz B, Griera M, Diez-Marqués L, Rodriguez-Puyol D et al. Nitric oxide regulates transforming growth factor-beta signaling in endothelial cells. Circ Res 2005; 97 (11): 1115-23.

64. Shifow AA, Kumar KV, Naidu MUR, Ratnakar KS. Melatonin, a pineal hormone with antioxidant property, protects against gentamicininduced nephrotoxicity in rats. Nephron 2000; 85 (2): 167-174.

65. Reiter RJ, Acuno-Castroviejo D, Tan DX, Burkhardt S. Free radical-mediated molecular damage, Ann. NY Acad Sci 2001; 939: 200-215.

66. Ross D. Glutathione, free radicals and chemotherapeutic agents. Pharmac Ther 1988; 37: 231-249.

67. Houghton DC, English J, Bennett WM. Chronic tubulointerstitial nephritis and renal insufficiency associated with longterm "subtherapeutic" gentamicin. J Lab Clin Med 1988; 112: 694-703.

68. Ozbek E, Cekmen M, Ilbey YO et al. Atorvastatin prevents gentamicin-induced renal damage in rats through the inhibition of $\mathrm{p} 38$-MAPK and NF-kB pathways. Renal Fail 2009; 31: 382-392. 